\title{
Primeros resultados en el desarrollo de un marcador genético basado en las proteínas de reserva en dos especies del género Nothofagus
}

\author{
First results in the development of a genetic marker based on the storage \\ proteins in two species of the Nothofagus genus
}

\author{
María Ángela Martína, Sonia Muñoz ${ }^{a, b}$, Fernando Muñoz ${ }^{c}$, Matilde Uribec, Juan Ramón Molina ${ }^{b}$, \\ Miguel Ángel Herrerab ${ }^{b}$, Luis Miguel Martín ${ }^{a}$, Juan Bautista Álvarez ${ }^{\text {** }}$
}

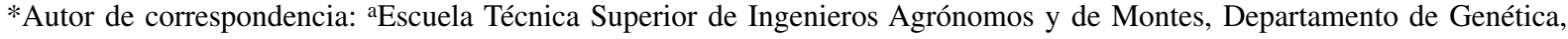
Córdoba, España, tel.: +34-957218505, fax: +34-957218503, jb.alvarez@uco.es

b Escuela Técnica Superior de Ingenieros Agrónomos y de Montes, Departamento de Ingeniería Forestal, Córdoba, España.

c Universidad de Concepción, Facultad de Ciencias Forestales, Departamento de Silvicultura, Concepción, Chile.
\end{abstract}

\begin{abstract}
SUMMARY
The Nothofagus genus (Nothofagaceae) in Chile includes 12 species and some hybrids among them. Some of these species are seriously threatened, presenting a low distribution and high genetic erosion. In this study a molecular marker based on seed storage proteins Nothofagus sp. was developed. Seed samples of Nothofagus alessandrii and N. glauca from two locations in southern Chile were analysed for each species. Among the different fractions of seed proteins (albumins, globulins, prolamins and glutelins) analyzed, the glutelins showed the best results, presenting conspicuous bands. Up to 39 bands were detected in both species; 22 in $N$. alessandrii (including 14 polymorphic) and 26 in $N$. glauca (21 polymorphic), with seven of them common to both species. There was a clear difference between the protein profiles of both species; we detected a preliminary value of genetic diversity in $N$. glauca greater than in $N$. alessandrii. These results, although preliminary, suggest that this marker could be useful for assessing genetic diversity in the Nothofagus genus.
\end{abstract}

Key words: electrophoresis, Nothofagus alessandrii, Nothofagus glauca, seed storage proteins.

\section{RESUMEN}

El género Nothofagus (Nothofagaceae) comprende 12 especies y algunos híbridos entre las mismas. Algunas de estas especies se encuentran seriamente amenazadas, presentando una distribución reducida y una gran erosión genética. En este estudio fue desarrollado un marcador molecular basado en las proteínas de reserva de la semilla de especies del género Nothofagus. Para ello se tomaron muestras de semilla de dos especies del género, $N$. alessandrii y $N$. glauca. De las diferentes fracciones de proteínas de la semilla (albúminas, globulinas, prolaminas y glutelinas), las glutelinas mostraron los mejores resultados al presentar bandas más conspicuas. Hasta 39 bandas fueron detectadas entre las dos especies, 22 en N. alessandrii (14 de ellas polimórficas) y 26 en $N$. glauca (21 polimórficas), siendo siete de ellas comunes a ambas especies. Se encontró una clara diferencia entre los perfiles proteicos de ambas especies, detectándose mayor diversidad genética en $N$. glauca que en $N$. alessandrii. Aunque estos resultados son preliminares, sugieren que este marcador podría ser útil para la evaluación de la diversidad genética en especies del género Nothofagus.

Palabras clave: electroforesis, Nothofagus alessandrii, Nothofagus glauca, proteínas seminales de reserva.

\section{INTRODUCCIÓN}

La diversidad genética de las especies es la base de su capacidad de respuesta ante los cambios que puedan suceder en su medio ambiente. Una especie con baja variabilidad genética será más vulnerable ante los cambios que se producen en los ecosistemas forestales, con lo que se incrementará el riesgo de extinción (Mace et al. 2003). Los sistemas de medida de la diversidad genética más empleados en la actualidad se basan en los marcadores moleculares. Estas moléculas pueden ser tanto ADN como moléculas que son el resultado directo de los genes (proteínas y metabolitos secundarios) que presentan regulación genética sencilla y para las que suele ser frecuente encontrar variación (Müller-Starck y Schubert 2000).

Uno de estos marcadores son las proteínas de reserva de la semilla, cuyo papel biológico es ser fuente de aminoácidos para los procesos de síntesis que tienen lugar durante la germinación. Esto ha propiciado que los genes codificantes de estas proteínas estén repetidos y sean neutros frente a la evolución, lo que conlleva un alto grado de variación. Además, estos marcadores son codominantes y pueden ser 
analizados mediante electroforesis, de forma muy económica (Gepts 1990). Se ha demostrado la posibilidad de usar estas proteínas en la evaluación de la diversidad genética de diversas especies forestales como: Castanea sativa Mill. (castaño) (Álvarez et al. 2003, Martín et al. 2005), Quercus ilex L. (encina) (Martín et al. 2009), Pinus pinea L. (pino piñonero) (Álvarez et al. 2004) y Abies pinsapo Boiss. (pinsapo) (Martín et al. 2008, 2010).

El género Nothofagus (familia Nothofagaceae) es el principal componente de los bosques templados del sur de Sudamérica, distribuyéndose entre los $32^{\circ}$ y $\operatorname{los} 56^{\circ} \mathrm{S}$ (Veblen et al. 1996). Dentro de este género pueden detectarse hasta 12 especies, junto con diversos híbridos naturales entre las mismas (Donoso 1987). Algunas de las especies del género como Nothofagus alessandrii Espinosa (ruil) y N. glauca (Phil.) Krasser (hualo) se encuentran seriamente amenazadas, siendo clasificadas como especie "en peligro de extinción" y especie "vulnerable", respectivamente (UICN 2001). Todo esto determina la necesidad de evaluar y caracterizar, a nivel genético, los rodales que aún se mantienen, con objeto de preservar su integridad genética y poder desarrollar estrategias que permitan su conservación.

En especies del género Nothofagus se han efectuado diversos trabajos con diferentes marcadores moleculares como isoenzimas o marcadores de ADN (Gallo et al. 2006, Acosta y Premoli 2010, Mathiasen y Premoli 2010). Sin embargo, sólo se tienen las referencias de Torres-Díaz et al. (2007) sobre isoenzimas de N. alessandrii y de Azpilicueta et al. (2004) sobre microsatélites (SSR) en N. glauca. No se ha encontrado ninguna referencia del uso de las proteínas de reserva de la semilla en el género.

En virtud de lo anterior, el objetivo es determinar las posibilidades de uso de las proteínas de reserva de la semilla como marcadores de la diversidad genética en estas dos especies del género Nothofagus.

\section{MÉTODOS}

Material vegetal. En un primer análisis, se tomaron 10 semillas viables de cada una de las especies motivo de estudio, con el fin de identificar la fracción proteica que almacenaban. A fin de tener una primera evaluación de la diversidad genética de ambas especies, se recogieron en torno a cinco semillas por árbol de dos localidades de cada una de ellas. Así, en el caso de $N$. alessandrii se dispuso de 209 semillas recogidas de la localidad de Curepto $\left(35^{\circ} 05^{\prime} 36^{\prime \prime} \mathrm{S} ; 72^{\circ} 01^{\prime} 01^{\prime \prime}\right.$ O) y 150 de la localidad de Empedrado ( $35^{\circ} 35^{\prime} 26^{\prime \prime} \mathrm{S} ; 71^{\circ} 33^{\prime} 11^{\prime \prime} \mathrm{O}$ ), ambas en la Cordillera de la Costa; mientras que en el caso de $N$. glauca se dispuso de 101 semillas recogidas en la Reserva de los Queules (35'58'55' S; 72 41' 13” O), en la Cordillera de la Costa, y 72 semillas de San Fabián de Alico ( $36^{\circ} 33^{\prime} 22^{\prime}$ ' S; $71^{\circ} 32^{\prime} 11^{\prime}$ " O), en la Cordillera de Los Andes.
Extracción de proteínas y análisis electroforético. Antes de la extracción de proteína, las muestras fueron deslipificadas con éter dietílico y acetona. Las proteínas fueron extraídas según el método secuencial descrito por Fonseca et al. (1997). Las cuatro fracciones obtenidas (albúminas, globulinas, prolaminas y glutelinas) fueron precipitadas con $1 \mathrm{~mL}$ de acetona fría y los pellets resuspendidos en $125 \mathrm{mM}$ Tris- $\mathrm{HCl} \mathrm{pH}: 6,8$ con $2 \%$ (p/v) dodecil sulfato de sodio (SDS), $10 \%(\mathrm{v} / \mathrm{v})$ glicerol, $0,02 \%(\mathrm{p} / \mathrm{v})$ azul de bromofenol y $2 \%(\mathrm{p} / \mathrm{v})$ ditiotreitol en relación 1:5 (p/v).

La separación electroforética se realizó en un sistema vertical en placas con condiciones desnaturalizantes (SDSPAGE) con un sistema de tampón discontinuo Tris-HCl-SDS (pH: 6,8-8,8) a una concentración de poliacrilamida del $12 \%$ (p/v, C: 2,67\%). Se utilizó el sistema de tampón Tris-HCl/ glicina indicado por Laemmli (1970). La electroforesis se realizó a una intensidad constante de $30 \mathrm{~mA} / \mathrm{gel}$ a $18^{\circ} \mathrm{C}$ hasta 20 minutos después de que el colorante trazador (azul de bromofenol) saliera del gel. Los geles fueron teñidos durante una noche con una solución de ácido tricloroacético al $12 \%(\mathrm{p} / \mathrm{v})$ con un $5 \%(\mathrm{v} / \mathrm{v})$ de etanol y $0,05 \%(\mathrm{p} / \mathrm{v})$ de azul brillante de Coomassie R-250. La decoloración del gel se llevó a cabo con agua corriente.

Nomenclatura y análisis estadístico. Las bandas fueron nombradas de manera consecutiva en orden de peso molecular descendente, independientemente de la especie en la que fueron detectadas, calculándose su frecuencia $\left(p_{i}\right)$ según presencia o ausencia. La diversidad genética fue estimada según el índice de Nei $-H e=1-\sum p_{i}^{2}$ (Nei 1972, 1973). Las distancias genéticas de Nei fueron utilizadas para generar un dendrograma según el método UPGMA (Sneath y Sokal 1973), validado mediante su valor cofenético (r) (Rohlf y Fisher 1968).

\section{RESULTADOS}

Uno de los principales problemas encontrados en el análisis de las semillas de Nothofagus spp. ha resultado ser el alto porcentaje de semillas vanas o severamente infectadas por hongos. Por ello, el número de semillas útiles para el análisis en cada una de las cuatro localidades mencionadas fueron 64 y 46 para N. alessandrii y 47 y 7 para $N$. glauca. Estos números tan reducidos, y especialmente el último, hicieron necesario reconducir el estudio de la diversidad de ambas especies a un nivel preliminar, en el que se ha valorado la posibilidad de utilización del marcador en ambas especies.

Las glutelinas fueron la única fracción útil en ambas especies, dado que las otras fracciones (albúminas, globulinas y prolaminas) o no mostraron bandas o éstas no fueron lo suficientemente conspicuas para poder ser evaluadas mediante los protocolos utilizados (figura 1).

En ambas especies, las zonas del gel más aptas para el estudio fueron la zona C, comprendida entre los $30 \mathrm{y}$ 


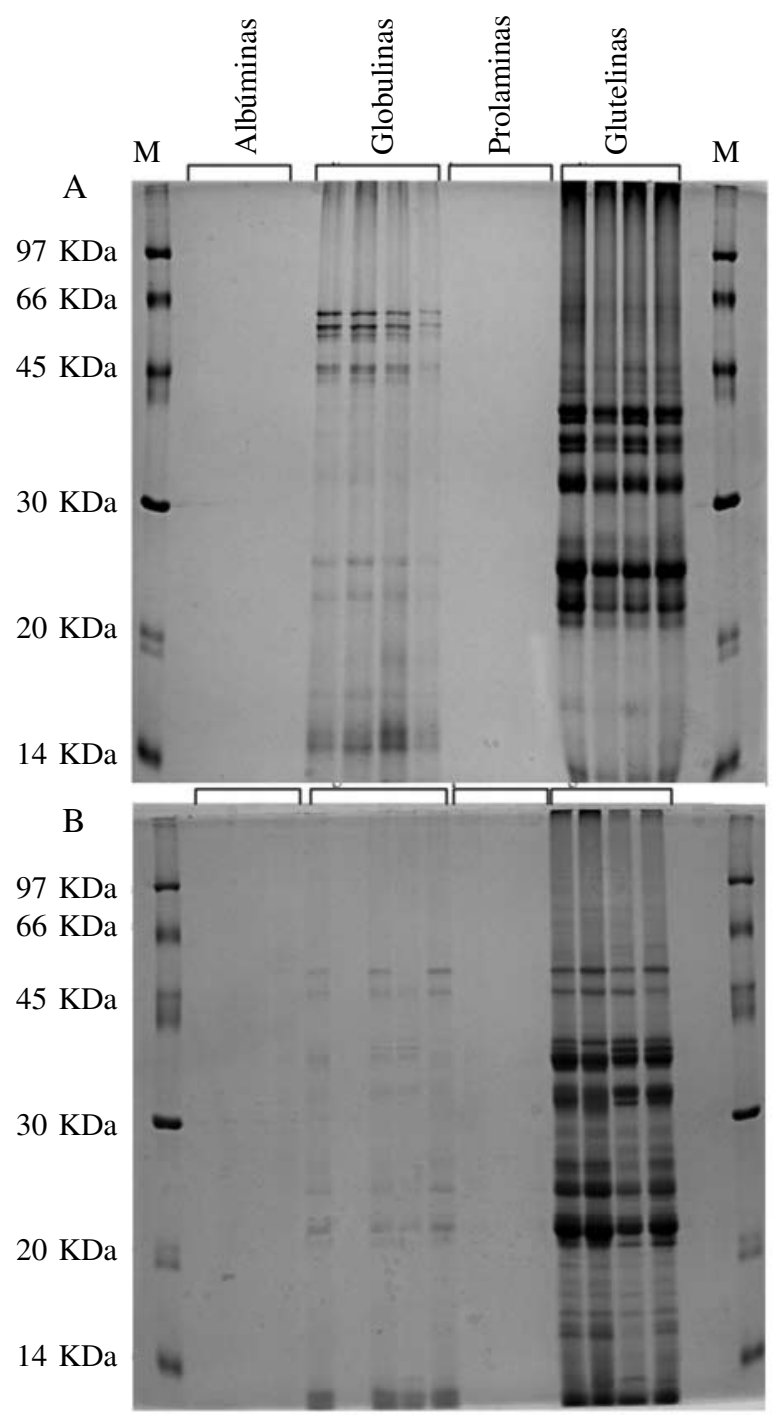

Figura 1. Gel al $12 \%$ de poliacrilamida en el que se muestran las cuatro fracciones proteicas (albúminas, globulinas, prolaminas y glutelinas) de Nothofagus alessandrii (A) y N. glauca (B). M: marcador molecular empleado como referencia.

Gel at $12 \%$ polyacrylamide concentration, where the four protein fractions (albumins, globulins, prolamins and glutelins) are shown in $N$. alessandrii (A) and $N$. glauca (B). M: molecular weight marker used as reference.

$45 \mathrm{KDa}$, y la zona D, entre los 20 y los $30 \mathrm{KDa}$. Este análisis preliminar ha permitido identificar un total de 39 bandas (20 para la zona C y 19 para la zona D) en el conjunto de las dos especies. En $N$. alessandrii se detectaron 12 bandas en la zona C y 10 en la zona $\mathrm{D}$, siendo polimórficas siete en cada una de las zonas (figura 2). En $N$. glauca se identificaron 12 bandas en la zona C y 14 en la zona $\mathrm{D}$, de las que fueron polimórficas 9 y 12 , respectivamente (figura 3). Los patrones de bandas de ambas especies fueron claramente diferentes, no obstante, cuatro bandas $(9 \mathrm{C}, 10 \mathrm{C}, 11 \mathrm{C}$ y $12 \mathrm{C}$ ) para la zona $\mathrm{C}$ y 3 bandas $(12 \mathrm{D}, 14 \mathrm{D}$ y $16 \mathrm{D})$ para la zona $\mathrm{D}$ fueron comunes para ambas especies (figuras 2 y 3 ).
Si bien, como previamente se ha indicado, el número de muestras fue muy bajo, se realizó una estimación de la diversidad genética de los materiales de ambas especies utilizados en el presente trabajo, agrupando las semillas de las dos localidades correspondientes a $N$. glauca. Los datos obtenidos indicaron que la diversidad genética de $N$. alessandrii $(H e=0,122)$ fue menor que la de $N$. glauca $(H e=0,241)$. El dendrograma basado en la matriz de distancias genética de Nei indicó que las diferencias entre las dos localizaciones de $N$. alessandrii fueron muy pequeñas, mientras que las diferencias entre especies son claras (figura 4).

\section{DISCUSIÓN}

El presente estudio muestra que las proteínas de reserva pueden ser utilizadas como sistema de estudio de la diversidad genética e hibridación de estas especies, siendo las gluteninas la fracción más adecuada para ello, coincidiendo con lo encontrado en encina por Martín et al. (2009). El análisis preliminar de la diversidad genética indica que el porcentaje de bandas polimórficas y, en general, la diversidad genética encontrada es superior en $N$. glauca frente a la encontrada en $N$. alessandrii. Este resultado es congruente con el menor rango de distribución y mayor amenaza de extinción en que se encuentra $N$. alessandrii.

Aunque no existen datos que permitan establecer comparaciones rigurosas, dado que este marcador no ha sido empleado en ninguna otra especie del género Nothofagus, los niveles de diversidad encontrados para estas proteínas, en particular en $N$. alessandrii, son superiores en comparación con los reportados por Torres-Díaz et al. (2007) usando isoenzimas, por lo que este estudio confirma el potencial de este marcador para el estudio de la diversidad genética.

Si bien el presente trabajo debe ser considerado como preliminar, abre una vía complementaria para la evaluación de la diversidad genética en especies del género Nothofagus, así como también en la detección de procesos de hibridación natural entre las especies del género, dadas las marcadas diferencias observadas en el perfil proteico de las especies evaluadas.

\section{AGRADECIMIENTOS}

Este trabajo ha sido financiado gracias al proyecto DIUC N ${ }^{\circ} 207.142 .029-1.0$ de la Dirección de Investigación de la Universidad de Concepción (Chile), así como a los proyectos (A/6387/06 y A/9485/07) de la Agencia Española de Cooperación Internacional para el Desarrollo, Ministerio de Asuntos Exteriores (España). Los autores agradecen al Dr. Rómulo Santelices de la Universidad Católica del Maule (Talca, Chile) por la entrega de muestras de ruil. 

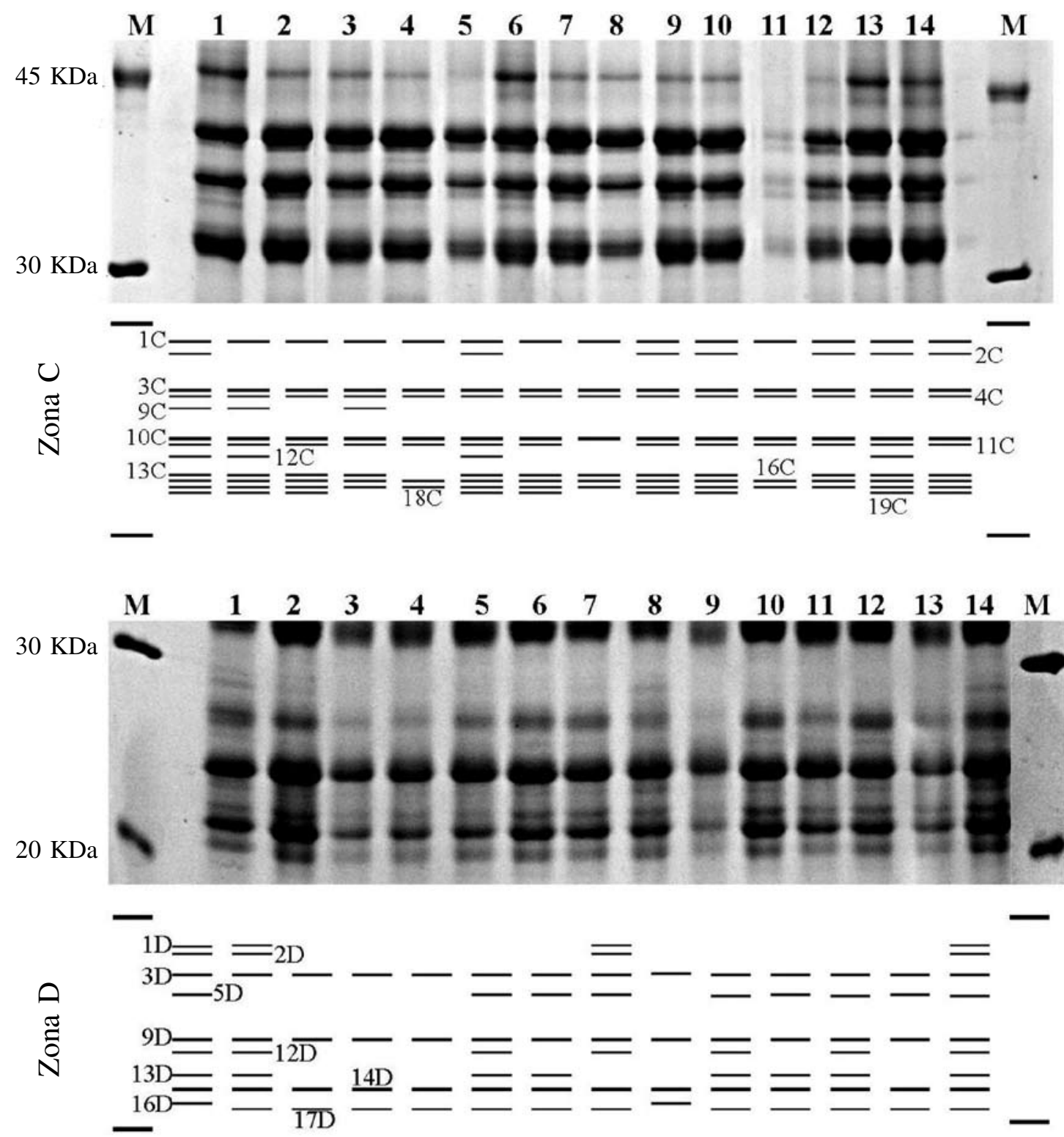

Figura 2. Gel y diagrama correspondiente a las bandas encontradas en las zonas C y D de Nothofagus alessandrii. Gel and diagram corresponding to the bands found in the $\mathrm{C}$ and $\mathrm{D}$ zones of $N$. alessandrii.

\section{REFERENCIAS}

Acosta MC, AC Premoli. 2010. Evidence of chloroplast capture in South American Nothofagus (subgenus Nothofagus, Nothofagaceae). Molecular Phylogenetics and Evolution 54: 235-242.

Álvarez JB, C Muñoz-Diez, A Martín-Cuevas, S López, LM Martín. 2003. Cotyledon storage proteins as markers of the genetic diversity in Castanea sativa Miller. Theoretical and Applied Genetics 107: 730-735.

Álvarez JB, MJ Toledo, B Abellanas, LM Martín. 2004. Use of megagametophyte storage proteins as markers of genetic diversity in stone pine (Pinus pinea L.) in Andalucia, Spain. Genetic Resources and Crop Evolution 51: 621-627.

Azpilicueta MM, H. Caron, C. Bodènés, L.A. Gallo. 2004. SSR markers for analysing South American Nothofagus species. Silvae Genetica 53: 240-243.
Donoso C. 1987. Variación natural en especies de Nothofagus en Chile. Bosque 8(2): 85-97.

Fonseca PA, RB Ferreira, AR Teixeira. 1997. Seed proteins from Quercus suber. Journal of Agricultural and Food Chemistry 45: 3443-3447.

Gallo LA, P Marchelli, MM Azpilicueta, P Crego. 2006. El uso de marcadores genéticos en el género Nothofagus con especial referencia a raulí y roble. Bosque 27 (1):3-15.

Gepts P. 1990. Genetic diversity of seed storage proteins in plants. In Brown AHD, MT Clegg, AL Kahler, BS Weir eds. Plant population: genetics, breeding, and genetic resources. Sunderland, Massachusetts, USA. Sinauer Associates. p. 64-82.

Laemmli UK. 1970. Cleavage of structural proteins during the assembly of the head of bacteriophage T4. Nature 227: 680-685. 

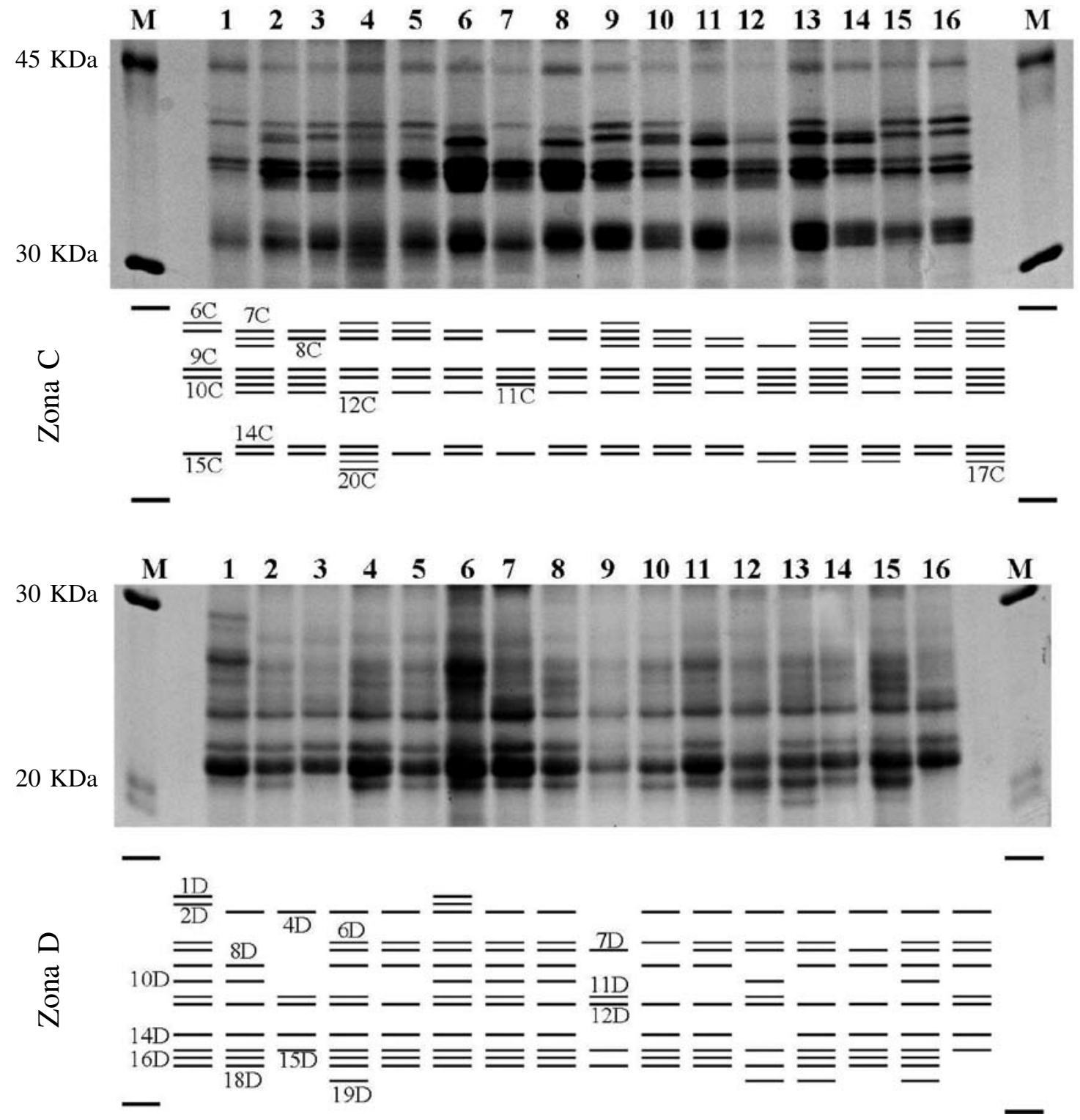

Figura 3. Gel y diagrama correspondiente a las bandas encontradas en las zonas C y D de Nothofagus glauca.

Gel and diagram corresponding to the bands found in the $\mathrm{C}$ and $\mathrm{D}$ zones of $N$. glauca.

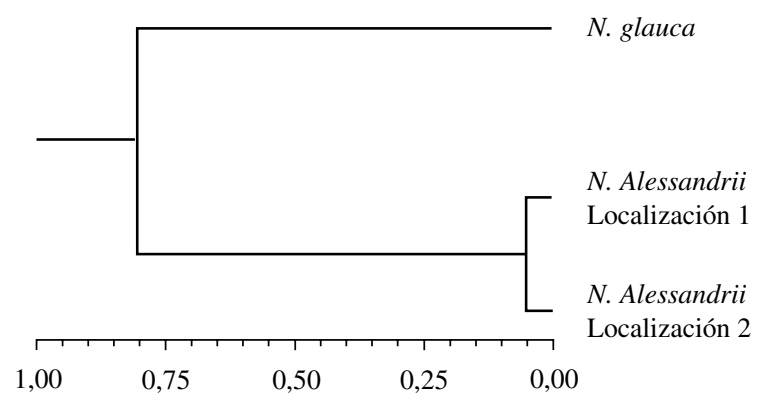

Figura 4. Dendrograma obtenido a partir de la matriz de distancias genéticas de Nei entre las localizaciones de Nothofagus alessandrii y $N$. glauca (correlación cofenética: $\mathrm{r}=0,991, P<0,01$ ).

Dendrogram based on Nei $=\mathrm{s}$ genetic distance matrix among the localizations of $N$. alessandrii and $N$. glauca (Co-phenetic correlation, $\mathrm{r}=0.991, P<0.01$ ).
Mace GM, JL Gittleman, A Purvis. 2003. Preserving the tree of life. Science 300: 1707-1709.

Martín MA, LM Martín, JB Álvarez. 2005. Cotyledon storage proteins in European sweet chestnut. Acta Horticulturae 693: 459-463.

Martín MA, JB Álvarez, LM Martín. 2010. Genetic diversity of Spanish fir (Abies pinsapo Boiss.) populations by means of megagametophyte storage proteins. Annals of Forest Science 67(603): 1-7.

Martín MA, LM Martín, JB Álvarez. 2008. Uso de las proteínas de reserva del megagametofito como marcador de la diversidad genética en Abies pinsapo. Cuadernos de la Sociedad Española de Ciencias Forestales 24: 63-66.

Martín MA, R Navarro-Cerrillo, P Ortega, JB Álvarez. 2009. The use of cotyledon proteins to assess the genetic diversity in sweet holm oak. Journal of Forest Science 55: 526-531. 
Mathiasen P, AC Premoli. 2010. Out in the cold: genetic variation of Nothofagus pumilio (Nothofagaceae) provides evidence for latitudinally distinct evolutionary histories in austral South America. Molecular Ecology 19:371-85.

Müller-Starck G, R Schubert. 2000. Genetic markers as tool for bioindication in forest ecosystem. In Young A, D Boshier, T Boyle eds. Forest conservation genetics. Principles and practice. Sydney, Australia. CSIRO-CABI Publishing. p. 227-237.

Nei M. 1972. Genetic distances between different populations. American Naturalist 106: 283-292.

Nei M. 1973. Analysis of gene diversity in subdivided populations. Proceedings of the National Academic Science USA 70: $3321-3323$

Rohlf FJ, DR Fisher. 1968. Test for hierarchical structure in random data sets. Systematic Zoology 17: 407-412.

Sneath PHA, RR Sokal. 1973. Numerical taxonomy Freeman, San Francisco, USA. 573 p.
Torres-Díaz C, E Ruiz, F González, G Fuentes, LA Cavieres. 2007. Genetic diversity in Nothofagus alessandrii (Fagaceae), an endangered endemic tree species of the Coastal Maulino Forest of Central Chile. Annals of Botany 100: 75-82.

UICN (Unión Internacional para la Conservación de la Naturaleza, $\mathrm{CH})$. 2001. Categorías y criterios de la lista roja de la UICN: Versión 3.1. Comisión de Supervivencia de Especies de la UICN. Gland, Suiza y Cambridge, Reino Unido. UICN. 33 p.

Veblen TT, C Donoso, T Kitzberger, AJ Reertus. 1996. Ecology of Southern Chilean and Argentinean Nothofagus forests. In Veblen TT, RS Hill, J Read eds. The ecology and biogeography of Nothofagus forests. New Haven, CT, USA. Yale University Press. p. 293-353.

Recibido: 16.06.10

Aceptado: 20.07.10 